\title{
OPERATORS COMMUTING WITH A COMPACT QUASIAFFINITY1
}

\author{
L. J. GRAY
}

ABSTRACT. We present an example of a nonbiquasitriangular operator which commutes with a compact quasiaffinity.

Let $\mathcal{H}$ denote a complex, separable Hilbert space, $B(\mathcal{H})$ the collection of bounded linear operators on $\mathcal{H}$. Recall that $X \in B(\mathcal{H})$ is called a quasiaffinity provided $X$ and $X^{*}$ have trivial null spaces; we will denote by $K_{a}$ the set of compact quasiaffinities. In a recent paper [2], Foias, Pearcy, and Voiculescu consider the problem of which operators can commute with an operator $K \in K_{a}$. They show that if $T$ is a nonbiquasitriangular operator such that $T K=K T$ and $K \in K_{a}$, then the index of $T-\lambda$ cannot be finite for any scalar $\lambda$ such that $T-\lambda$ is a semi-Fredholm operator. To complete the discussion, they give an example (Proposition 5.3) of a semi-Fredholm operator $T$ with infinite index and $K \in K_{a}$ such that $T$ and $K$ commute. However, this example is based upon the incorrect statement that the Volterra operator $V$ is similar to $V / 2$ (see [3]). In fact, it follows from a result of Kalisch [4, Lemma 5], that if $X \in B(\mathcal{H})$ is similar to $X / 2$, then $X$ must be nilpotent. Thus, there is no compact quasiaffinity which can replace the Volterra operator in their example.

In this note, we show that a slight modification of the construction presented in [2] can be used to provide the desired example. We define the semi-Fredholm operator $T$ and the operator $K \in K_{a}$ on the Hilbert space $\mathcal{H} \oplus \mathcal{H} \oplus \mathcal{H} \oplus \cdots$ via the operator matrices

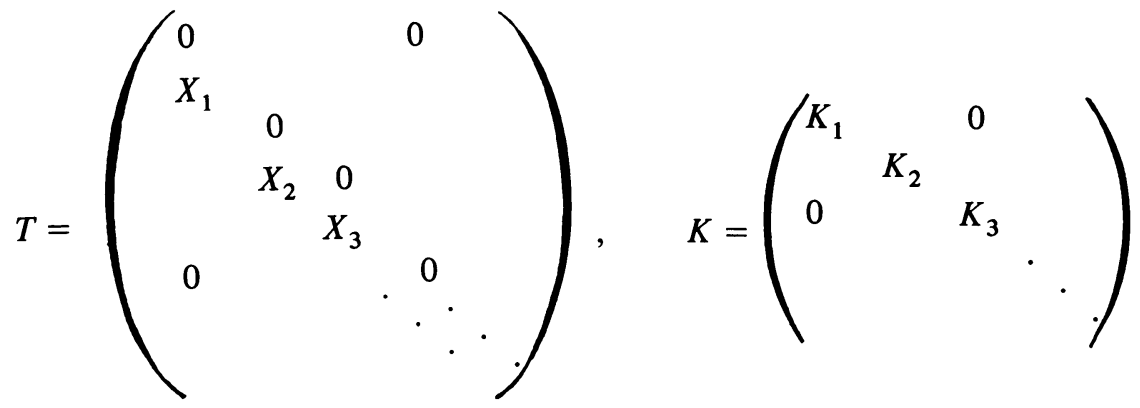

Received by the editors September 17, 1976.

AMS (MOS) subject classifications (1970). Primary 47B99; Secondary 47B05, 47B30.

Key words and phrases. Compact quasiaffinity, semi-Fredholm, weighted shift.

1 This work was supported by the NSF as part of the Summer Institute on Operator Theory held at the University of New Hampshire. 
In order for $T$ to be semi-Fredholm, it is sufficient that $X_{j}$ be invertible for all $j$, and $\sup _{j}\left\{\left\|X_{j}\right\|,\left\|X_{j}^{-1}\right\|\right\}<\infty$. Furthermore, if $K_{j} \in K_{a}$ for all $j$, and $\left\|K_{j}\right\|$ $\rightarrow 0$, then $K \in K_{a}$. Finally, a computation shows that $T$ and $K$ commute provided $K_{j+1}=X_{j} K_{j} X_{j}^{-1}$.

Let $\left\{e_{m}\right\}, m=0, \pm 1, \pm 2, \ldots$, be an orthonormal basis for $\mathcal{H}$. We define $K_{1}$ to be a compact bilateral weighted shift on $\mathcal{H}$; i.e., $K_{1} e_{m}=w_{1, m} e_{m+1}$, where $w_{1, m}>0$ and $w_{1, m} \rightarrow 0$. Thus, $K_{1} \in K_{a}$. Since $K_{1}$ is compact, $\lim _{m \rightarrow \pm \infty} w_{1, m}$ $=0$, and thus there exists an index $m_{0}$ such that $w_{1, m_{0}}=\max _{m}\left\{w_{1, m}\right\}$. We now define $K_{2} \in K_{a}$ via $K_{2} e_{m}=w_{2, m} e_{m+1}$, where $w_{2, m}=w_{1, m}$ for $m \neq m_{0}$, and $w_{2, m_{0}}=w_{1, m_{0}} / 2$. Theorem 2 of [5] says that $K_{1}$ and $K_{2}$ are similar, and if $K_{2} \stackrel{2}{=} X_{1} K_{1} X_{1}^{-1}$, then $X_{1}$ can be chosen such that $\max \left\{\left\|X_{1}\right\|,\left\|X_{1}^{-1}\right\|\right\} \leqslant 2$. If we now define $K_{j+1}$ from $K_{j}$ in the same fashion, then clearly $\left\|K_{j}\right\| \rightarrow 0$ and $\sup _{j}\left\{\left\|X_{j}\right\|,\left\|X_{j}^{-1}\right\|\right\} \leqslant 2$. Hence, Proposition 5.3 of [2] is established.

As a final comment, we point out that a result of R. G. Douglas [1, Theorem 8] shows that $T$ cannot be an isometry.

\section{REFERENCES}

1. R. G. Douglas, On the operator equation $S^{*} X T=X$ and related topics, Acta Sci. Math. (Szeged) 30 (1969), 19-32. MR 40 \#334.

2. C. Foias, C. Pearcy and D. Voiculescu, On the staircase representation of biquastriangular operators, Michigan Math. J. 22 (1975), 343-352.

3. C. Foias and J. P. Williams, Some remarks on the Volterra operator, Proc. Amer. Math. Soc. 31 (1972), 177-184. MR 45 \#4194.

4. G. Kalisch, On similarity, reducing manifolds, and unitary equivalence of certain Volterra operators, Ann. of Math. (2) 66 (1957), 481-494. MR 19, 970.

5. A. Shields, Weighted shift operators and analytic function theory, Topics in Operator Theory, Math. Surveys, No. 13, Amer. Math. Soc., Providence, R.I., 1974, pp. 49-128. MR 50 \# 14341.

Union Carbide Corporation, Nuclear Division, Oak Ridge, Tennessee 37830 